\title{
An Exact Analytical Solution to the Static Cracks in Finite-width Single-edge Cracked Strips
}

\author{
Lu Guan ${ }^{1, a^{*}}$ \\ ${ }^{1}$ School of Statistics and Mathematics, Inner Mongolia Finance and Economics \\ University, Hohhot 010070, China \\ aguanlusxy@126.com
}

Keywords: finite-width single-edge cracked strip, static crack, conformal mapping, stress intensity factor

Abstract. Using the method of complex analysis and through constructing new conformal mapping functions, we analyzed the plane elasticity problem of static cracks in finite-width single-edge cracked strips, and provided an exact analytical solution to the crack-tip stress intensity factor.

\section{Introduction}

As of present, there have been various studies regarding finite-height crack strips of different materials. Article [1], for instance, provides an analytical solution to two semi-infinite collinear crack strips; article [2] provides an analytical solution to Type II cracks in piezoelectric ceramic strips. Article [3] provides a conformal mapping function and conformal mapped the finite-width single-edged crack strip to the upper half plane, from which the stress intensity factors (SIFs) $K_{\square}, K_{\square}$ of static cracks are obtained. In this paper we have further analyzed and improved the results of article [3] by constructing new conformal mapping functions, and have obtained the exact analytical solution to crack tip SIFs.

\section{The Static Crack Problem in Finite-width Single-edge Cracked Tips}

Let there be a single crack in a finite-with strip, under plane stress or plane strain state as shown in figure 1 . The governing equation for this problem is ${ }^{[4]}$

$$
\nabla^{2} \nabla^{2} U=0
$$

in which $\nabla^{2}$ is the two-dimension Laplace operator.

$$
U(x, y)=\operatorname{Re}\left[\bar{z} \phi_{1}(z)+\int \psi_{1}(z) d z\right]
$$

In which $\phi_{1}(z)$ and $\psi_{1}(z)$ represent two analytic functions of the complex variable $z=x+\mathrm{i} y$, $\bar{z}=x-\mathrm{i} y$ is the complex conjugate of $z$, Re represents real part of the complex number. From the fundamentals of electrostatics and equation (2), we have the following expression of stress and displacement

$$
\begin{aligned}
& \left\{\begin{array}{l}
\sigma_{x x}+\sigma_{y y}=4 \operatorname{Re} \phi_{1}^{\prime}(z) \\
\sigma_{y y}-\sigma_{x x}+2 \mathrm{i} \sigma_{x y}=2\left[\bar{z} \phi_{1}^{\prime \prime}(z)+\psi_{1}^{\prime}(z)\right]
\end{array}\right. \\
& 2 \mu\left(u_{x}+\mathrm{i} u_{y}\right)=\kappa \phi_{1}(z)-z \overline{\phi_{1}^{\prime}(z)}-\overline{\psi_{1}(z)}
\end{aligned}
$$

in which $\kappa= \begin{cases}(3-v) /(1+v), & \text { plane stress } \\ 3-4 v, & \text { plane strain }\end{cases}$ 


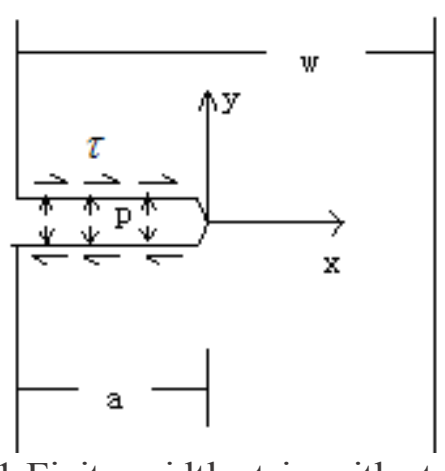

Fig. 1 Finite-width strip with static crack In this study we construct a new conformal mapping function

$$
z=\omega(\zeta)=\left(\frac{2 w}{\pi}\right) \arctan \left[\sqrt{\tan ^{2} \frac{\pi a}{2 w}-\zeta^{2}}\right]-a
$$

The conformal mapping function equation (5) maps the upper half plane (mathematical plane) of the $\zeta(=\xi+i \eta)$ plane, shown in figure 2, to the $z$ plane (mathematical plane) of the strip region of strip cracks, shown in figure 1 . So that crack tip $z=0$ corresponds to $\zeta=0$, and $z=-a^{+}$(upper part of the crack) corresponds to $\zeta=-\tan \frac{\pi a}{2 w}, z=-a^{-}$(lower part of the crack) corresponds to $\zeta=\tan \frac{\pi a}{2 w}$

Under transformation equation (5), functions $\phi_{1}(z)$ and $\psi_{1}(z)$ as well as their derivatives are transformed to

$$
\begin{cases}\phi_{1}(z)=\phi_{1}[\omega(\zeta)]=\phi(\zeta), & \psi_{1}(z)=\psi_{1}[\omega(\zeta)]=\psi(\zeta) \\ \phi_{1}^{\prime}(z)=\phi^{\prime}(\zeta) / \omega^{\prime}(\zeta), & \psi_{1}^{\prime}(z)=\psi^{\prime}(\zeta) / \omega^{\prime}(\zeta)\end{cases}
$$

When the crack plane is under uniform internal pressure (Type I problem), we have the following boundary conditions

$$
\left\{\begin{array}{cccc}
\sigma_{y y}=\sigma_{x y}=0, & y= \pm \infty, & -a<x<w-a & \\
\sigma_{x x}=\sigma_{x y}=0, & x=-a, & x=w-a, & -\infty<y<\infty \\
\sigma_{y y}=f_{1}(x), & \sigma_{x y}=0, & y= \pm 0, & -a<x<0
\end{array}\right.
$$

in which $f_{1}(x)$ is a known arbitrary function, here $f_{1}(x)=-p=$ constant. Therefore, the solution to the problem comes down to solving the following equation set ${ }^{[5]}$

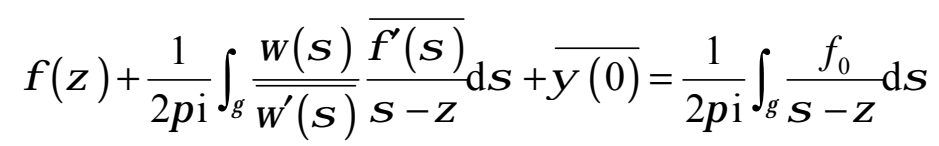

$$
\begin{aligned}
& \psi(\zeta)+\frac{1}{2 \pi \mathrm{i}} \int_{\gamma} \frac{\overline{\omega(\sigma)}}{\omega^{\prime}(\sigma)} \frac{\phi^{\prime}(\sigma)}{\sigma-\zeta} \mathrm{d} \sigma+\overline{\phi(0)}=\frac{1}{2 \pi \mathrm{i}} \int_{\gamma} \frac{\overline{f_{0}}}{\sigma-\zeta} \mathrm{d} \sigma
\end{aligned}
$$

in which

$$
f_{0}=\mathrm{i} \int(\bar{X}+\mathrm{i} \bar{Y}) \mathrm{d} s=\left\{\begin{array}{cc}
-p(z+a), & z \in A \Theta B \\
0, & z \notin A \Theta B
\end{array}\right.
$$


Note

$$
F(\sigma)=\frac{\omega(\sigma)}{\overline{\omega^{\prime}(\sigma)}} \overline{\phi^{\prime}(\sigma)}
$$

then

$$
\begin{aligned}
& F(\zeta)=\frac{\omega(\zeta)}{\overline{\omega^{\prime}(\bar{\zeta})}} \overline{\phi^{\prime}(\bar{\zeta})} \\
& =\frac{\left[\frac{\pi a}{2 w}-\arctan \left(\sqrt{\tan ^{2} \frac{\pi a}{2 w}-\zeta^{2}}\right)\right]\left[\left(1+\tan ^{2} \frac{\pi a}{2 w}-\zeta^{2}\right) \sqrt{\tan ^{2} \frac{\pi a}{2 w}-\zeta^{2}}\right] \frac{}{\zeta} \phi^{\prime}(\bar{\zeta})}{\zeta}
\end{aligned}
$$

Therefore $F(\zeta)$ is the analytic function on the lower half plane.

From the stress-free condition at infinity we get ${ }^{[6]}$

$$
\lim _{z \rightarrow \infty} z \overline{\phi^{\prime}(z)}=0
$$

Then from equation (5) and equation (13) we get

$$
\lim _{\zeta \rightarrow \infty} F(\zeta)=\lim _{\zeta \rightarrow \infty} \frac{\omega(\zeta)}{\overline{\omega^{\prime}(\bar{\zeta})}} \overline{\phi^{\prime}(\bar{\zeta})}=\lim _{z \rightarrow \infty} z \overline{\phi^{\prime}(z)}=0
$$

Using the Canchy intergral formula on the straight line we get

$$
\frac{1}{2 \pi \mathrm{i}} \int_{r} \frac{F(\sigma)}{\sigma-\zeta} \mathrm{d} \sigma=F(\infty)=0
$$

Then from equations(5), (10), (15), the equation (8) can become

$$
\phi(\zeta)+\overline{\psi(0)}=\frac{1}{2 \pi \mathrm{i}} \int_{-\tan \frac{\pi a}{2 w}}^{\tan \frac{\pi a}{2 w}} \frac{-p[\omega(\sigma)+a]}{\sigma-\zeta} \mathrm{d} \sigma
$$

Similarly, $\psi(\zeta)$ can also be determined from solving equation (9). Below we will calculate the vital physical quantity - the stress intensity factor ${ }^{[5]}$

$$
K_{\square-} \mathrm{i} K_{\square}=2 \sqrt{\pi} \lim _{\zeta \rightarrow 0} \frac{\phi^{\prime}(\zeta)}{\sqrt{\omega^{\prime \prime}(\zeta)}}=2 \sqrt{\pi} \frac{\phi^{\prime}(0)}{\sqrt{\omega^{\prime \prime}(0)}}
$$

Using integration by parts we obtain from equation (16)

$$
\phi^{\prime}(\zeta)=\frac{-p}{2 \pi \mathrm{i}} \int_{-\tan \frac{\pi a}{2 w}}^{\tan \frac{\pi a}{\omega}} \frac{\omega^{\prime}(\sigma)}{\sigma-\zeta} \mathrm{d} \sigma
$$

From equation (5) we get

$$
\omega^{\prime}(\zeta)=\left(\frac{2 w}{\pi}\right) \frac{-\zeta}{\left(1+\tan ^{2} \frac{\pi a}{2 w}-\zeta^{2}\right) \sqrt{\tan ^{2} \frac{\pi a}{2 w}-\zeta^{2}}}
$$

Substituting equation (19) into equation (18), we get 


$$
\begin{aligned}
& \phi^{\prime}(0)=\frac{p w}{\pi^{2} i} \lim _{\zeta \rightarrow 0} \int_{-\tan \frac{\pi a}{2 w}}^{\tan \frac{\pi a}{2 w}} \frac{\sigma}{\sqrt{\tan ^{2} \frac{\pi a}{2 w}-\sigma^{2}}(\sigma-\zeta)\left(1+\tan ^{2} \frac{\pi a}{2 w}-\sigma^{2}\right)} \mathrm{d} \sigma \\
& =\frac{-\mathrm{i} p w}{\pi^{2}} \lim _{x \rightarrow \tan \frac{\pi a}{2 w}} \frac{1}{\sqrt{1+x^{2}}}\left(\arctan \frac{\tan \frac{\pi a}{2 w} \sqrt{1+x^{2}}-x^{2}}{\left.\sqrt{x^{2}-\tan ^{2} \frac{\pi a}{2 w}}+\arctan \frac{\tan \frac{\pi a}{2 w} \sqrt{1+x^{2}}+x^{2}}{\sqrt{x^{2}-\tan ^{2} \frac{\pi a}{2 w}}}\right)}\right. \\
& =\frac{p w}{\pi i} \cos \frac{\pi a}{2 w}
\end{aligned}
$$

In the above equation we have already taken $\arctan (+\infty)=\frac{\pi}{2}$.

From equation (5) we can get

$$
\omega^{\prime \prime}(0)=-\frac{2 w}{\pi} c \tan \frac{\pi a}{2 w} \cdot \cos ^{2} \frac{\pi a}{2 w}
$$

Substituting equation (20), (21) into equation (17) and simplify to get $K_{\square}-\mathrm{i} K_{\square}=\sqrt{2} p \sqrt{w \tan \frac{\pi a}{2 w}}$ Then

$$
K_{\square}=\sqrt{2} p \sqrt{w \tan \frac{\pi a}{2 w}}
$$

The changing curve of $K_{\square}$ following parameters p , a , w is shown in figure 3.

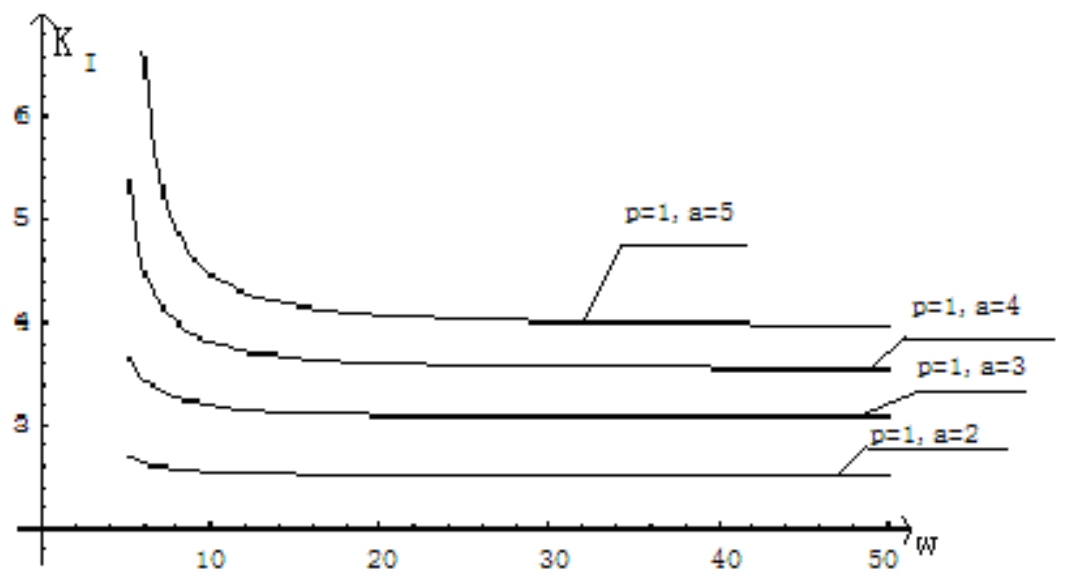

Figure 3 Changing curve of the crack tip SIF following parameters $\mathrm{p}, \mathrm{a}, \mathrm{w}$

Similarly, when crack surface is under uniform shear and the shearing strength is $-\tau$, we can obtain

$$
K_{\square}=\sqrt{2} \tau \sqrt{w \tan \frac{\pi a}{2 w}}
$$

Through constructing new conformal mapping, our study re-derived the static stress intensity factor of finite-width single-edged crack strips. It is slightly different from the results of article [3]. After careful reading we have found errors within some of the steps in article [3], such as those that would have influenced the calculation of results: the original equation, $\phi^{\prime}(0)=\frac{p a}{\pi i} \sin \frac{\pi a}{2 w}$, should have been $\phi^{\prime}(0)=\frac{p w}{\pi \mathrm{i}} \sin \frac{\pi a}{2 w}$ (equation(22)). Calculating with the corrected results, the stress intensity factor expression is identical to the conclusion of our study. 


\section{Conclusions}

Regarding the static crack problem in finite-width single-edge cracked strips, the conformal mapping equation (5) provided by this article is a transcendental function, using conformal mapping to simplify the complicated crack problem in order to obtain a solution, and the calculation method is relatively simple. The method in this article is an extension to the Muskhelishvili[ ${ }^{[4]}$ complex potential method, expanding the scope of application and enriching the content of the latter. An exact analytical solution to the static crack in finite-width single-edge cracked strips also provides great significance in solving many practical problems in engineering fracture.

\section{References}

[1] Dawe Shen, Tianyou Fan, Exact solutions of two semi-infinite collinear cracks in a strip, Engineering Fracture Mechanics. 70(2003) 813-822.

[2] Xianfang Li, Tianyou Fan, provides an analytical solution to Type III cracks in piezoelectric ceramic strips, Acta Mechanica Sinica. 34(2002) 335-343.

[3] Tianyou Fan, Xiaochun Yang, Hexin Li.Exact Analytic Solutions for a Finite Width a Single Edge Crack, CHIN.PHYS.LETT.16(1999) 32-34.

[4] Muskhelishvili NI, Some Basic Problems of Mathematical Theory of Elasticity, P Noordhoff, Holland, 1953.

[5] Tianyou Fan, Foundation of Fracture Theory, Science Press, Beijing, 2003.

[6] Zhilun Xu, Elastic Mechanics, Higher Education Press, Beijing, 1990. 